\title{
On Dual-Complex Numbers with Generalized Fibonacci and Lucas Numbers Coefficients
}

\author{
Arzu Cihan, Ayşe Zeynep Azak and Mehmet Ali Güngör*
}

\begin{abstract}
In this paper, dual-complex Fibonacci numbers with generalized Fibonacci and Lucas coefficients are defined. Generating function is given for this number system. Binet's formula is obtained by the help of this generating function. Then, well-known Cassini, Catalan, d'Ocagne's, Honsberger, Tagiuri and other identities are given for this number system. Finally, it is seen that the theorems and the equations which are obtained for the special values $p=1$ and $q=0$ correspond to the theorems and identities in [2].
\end{abstract}

Keywords: Dual-complex numbers; generalized Fibonacci numbers; generalized Lucas numbers.

AMS Subject Classification (2020): Primary: 11B39 ; Secondary: $11 B 83$.

${ }^{*}$ Corresponding author

\section{Introduction}

In the 12th century, Fibonacci number sequence was introduced in Liber Abaci which was written by Italian mathematician Leonardo Fibonacci. Then, this number sequence became important from three fundamental reasons. Firstly, the elements of the sequence appear in many places of nature such as plants and insects. Secondly, we get nearly the same rate when we divide any two successive Fibonacci numbers of the sequence. This number, called the "golden ratio", appears in numerous fields from the proportion of the human body to the pyramids of Egypt. Thirdly, there are many interesting features in the theory of these numbers. This number sequence is obtained by adding two consecutive terms, the first two terms being 0 and 1 . If the initial values are changed, another number sequence is created that is completely independent from the Fibonacci number sequence. With this in mind, the French mathematician Edward Lucas defined the Lucas number sequence by using the initial values 2 and 1, respectively and this number sequence gained serious popularity, too. The fact that the Lucas number sequence is so widely studied in the literature that is there are many interesting identities between the Fibonacci number sequence and this sequence.

Numerous studies have been done on Fibonacci and Lucas number sequences $[1,3,10]$. One of these studies is on dual-complex numbers with Fibonacci and Lucas numbers. Dual-complex numbers with Fibonacci and Lucas coefficients are defined in [2]. In this paper, we define dual-complex numbers by using the coefficients of generalized Fibonacci and Lucas numbers. Then the addition, multiplication, conjugation and modules of these numbers have been presented and identities have been obtained related to them. Also, the generating function of the dual-complex number with generalized Fibonacci coefficient has been obtained and the Binet formula for this number is proved. Finally, d'Ocagne's, Honsberger, Tagiuri, Catalan identities have been found and Cassini's identity has been given in case of $r=1$.

\section{Preliminaries}

Fibonacci and Lucas numbers are defined by the following recurrence relations

$$
F_{0}=0, \quad F_{1}=1, \ldots, \quad F_{n+1}=F_{n}+F_{n-1}, \quad n \geq 1
$$

Received : 18-09-2019, Accepted : 26-02-2020 
and

$$
L_{0}=2, \quad \mathrm{~L}_{1}=1, \ldots, \quad \mathrm{L}_{n+1}=L_{n}+L_{n-1}, \quad n \geq 1
$$

where $F_{n}$ and $L_{n}$ are the $n$-th Fibonacci and Lucas numbers, respectively. Also, Binet formula for the $n$-th Fibonacci and Lucas numbers are given by

$$
F_{n}=\frac{1}{\sqrt{5}}\left(\alpha^{n}-\beta^{n}\right) \quad \text { and } \quad L_{n}=\alpha^{n}+\beta^{n}, \quad n \geq 1
$$

(see ref. [9]) .

In 1961 Horadam defined the generalized Fibonacci numbers by using Fibonacci numbers such as

$$
H_{1}=p, \quad H_{2}=p+q, \quad p, q \in Z
$$

the recurrence relation is given by

$$
H_{n}=H_{n-1}+H_{n-2}, \quad n \geq 3
$$

and

$$
H_{n}=(p-q) F_{n}+q F_{n+1} .
$$

In this last equation, if we take $p=1$ and $q=0$, then the generalized Fibonacci number corresponds to Fibonacci number. If we take $p=1$ and $q=2$, then the generalized Lucas number corresponds to Lucas number. Also, Horadam defined Binet formula for the $n$-th generalized Fibonacci number as follows

$$
H_{n}=\frac{1}{2 \sqrt{5}}\left(\alpha^{n}-\mu \beta^{n}\right)
$$

(see ref. [4]).

On the other hand, Majernik has initially introduced dual-complex numbers, [7]. Then, Messelmi has given the algebraic properties of dual-complex numbers, [8]. The set of dual-complex numbers has been defined by

$$
D C=\left\{w=z_{1}+z_{2} \varepsilon \mid z_{1}, z_{2} \in C \text { where } \varepsilon^{2}=0, \varepsilon \neq 0\right\} .
$$

Multiplication scheme of these basis elements is shown by follows:

Table 1. Multiplication scheme of the dual-hyperbolic units.

\begin{tabular}{c|c|c|c|c}
$\times$ & 1 & $i$ & $\varepsilon$ & $i \varepsilon$ \\
\hline 1 & 1 & $i$ & $\varepsilon$ & $i \varepsilon$ \\
\hline$i$ & $i$ & -1 & $i \varepsilon$ & $-\varepsilon$ \\
\hline$\varepsilon$ & $\varepsilon$ & $i \varepsilon$ & 0 & 0 \\
\hline$i \varepsilon$ & $i \varepsilon$ & $-\varepsilon$ & 0 & 0
\end{tabular}
as

If we take complex numbers as $z_{1}=x_{1}+x_{2} i$ and $z_{2}=y_{1}+y_{2} i$, then any dual-complex number can be written

$$
w=x_{1}+x_{2} i+y_{1} \varepsilon+y_{2} i \varepsilon .
$$

There exists five different conjugates for this numbers and these conjugates can be given as follows:

$$
\begin{aligned}
& |\omega|^{\dagger_{1}}=\bar{z}_{1}+\bar{z}_{2} \varepsilon \quad \text { complex conjugation, } \\
& |\omega|^{\dagger_{2}}=z_{1}-z_{2} \varepsilon \quad \text { dual conjugation, } \\
& |\omega|^{\dagger_{3}}=\bar{z}_{1}-\bar{z}_{2} \varepsilon \quad \text { coupled conjugation, } \\
& |\omega|^{\dagger_{4}}=\bar{z}_{1}\left(1-\frac{z_{2}}{z_{1}} \varepsilon\right) \quad(\omega \in D C-A) \quad \text { dual - complex conjugation, } \\
& |\omega|^{\dagger_{5}}=z_{2}-z_{1} \varepsilon \quad \text { anti - dual "conjugation, }
\end{aligned}
$$

where "- " denotes the standard complex conjugation and the zero divisors of $D C$ is defined by the set $A$, [8]. Namely, $D C-A$ is a multiplicative group. The dual complex numbers form a commutative ring with 0 characteristic. Unlike quaternions, the multiplication of dual-complex numbers with generalized Fibonacci and Lucas number has a commutative ring structure. At the same time, the multiplication of dual-complex numbers with generalized Fibonacci and Lucas number gives two-dimensional complex Clifford and 4-dimensional real Clifford algebra structure. 


\section{Properties of Dual-Complex Numbers with Generalized Fibonacci and Lucas Coefficients}

Dual-complex Fibonacci and Lucas numbers are defined by

$$
D C F_{n}=F_{n}+F_{n+1} i+F_{n+2} \varepsilon+F_{n+3} i \varepsilon
$$

and

$$
D C L_{n}=L_{n}+L_{n+1} i+L_{n+2} \varepsilon+L_{n+3} i \varepsilon,
$$

respectively. Here $F_{n}$ and $L_{n}$ are the $n$-th Fibonacci and Lucas numbers, respectively and $\varepsilon$ denotes dual unit, $i$ denotes imaginary unit $\left(i^{2}=-1\right)$, $i \varepsilon$ denotes imaginary dual unit $\left(i \varepsilon^{2}=0\right)$. These numbers have been identified in [2]. Modules, conjugates and some well-known identities for Fibonacci and Lucas numbers have been obtained for them. Then, the correspondence of Binet formula for Fibonacci and Lucas numbers have been given. Also, d'Ocagne's, Honsberger, Tagiuri, Cassini and Catalan identities for these numbers have been found.

Now, let's define dual-complex Fibonacci numbers with generalized Fibonacci and Lucas coefficients in the light of paper [2].

Definition 3.1. $H_{n}$ is called generalized Fibonacci number which has recurrence relation such as

$$
H_{n}=H_{n-1}+H_{n-2} \quad \text { or } \quad H_{n}=(p-q) F_{n}+q F_{n+1}, \quad n \geq 3
$$

with the initial conditions

$$
H_{1}=p, \quad H_{2}=p+q, \quad H_{3}=2 p+q, \ldots \quad p, q \in Z .
$$

In that case, the set of generalized Fibonacci and Lucas number sequences are defined by

$$
D C X=\left\{D C X_{n}=R_{n}+R_{n}^{*} \varepsilon=\left(H_{n}+H_{n+1} i\right)+\left(H_{n+2}+H_{n+3} i \varepsilon\right) \mid H_{n} \text { is generalized Fibonacci number }\right\}
$$

and

$$
D C Y=\left\{D C Y_{n}=P_{n}+P_{n}^{*} \varepsilon=\left(V_{n}+V_{n+1} i\right)+\left(V_{n+2}+V_{n+3} i \varepsilon\right) \mid V_{n} \text { is generalized Lucas number }\right\}
$$

where $\varepsilon$ is dual unit, $i$ is imaginary unit and $i \varepsilon$ is imaginary dual unit. Hence, basis elements of the dual-complex numbers systems with generalized Fibonacci and Lucas coefficients are denoted by $(1, i, \varepsilon, i \varepsilon)$.

Let $D C X_{n}^{1}$ and $D C X_{n}^{2}$ denote the dual-complex numbers with generalized Fibonacci coefficients. These numbers are given by

$$
D C X_{n}^{1}=R_{n}^{1}+R_{n}^{1 *} \varepsilon=H_{n}^{1}+H_{n+1}^{1} i+H_{n+2}^{1} \varepsilon+H_{n+3}^{1} i \varepsilon
$$

and

$$
D C X_{n}^{2}=R_{n}^{2}+R_{n}^{2 *} \varepsilon=H_{n}^{2}+H_{n+1}^{2} i+H_{n+2}^{2} \varepsilon+H_{n+3}^{2} i \varepsilon
$$

respectively.

Addition, substraction and multiplication of these numbers are defined by

$$
\begin{aligned}
D C X_{n}^{1} \pm D C X_{n}^{2} & =\left(R_{n}^{1}+R_{n}^{1 *} \varepsilon\right) \pm\left(R_{n}^{2}+R_{n}^{2 *} \varepsilon\right) \\
& =\left(H_{n}^{1}+H_{n+1}^{1} i+H_{n+2}^{1} \varepsilon+H_{n+3}^{1} i \varepsilon\right) \pm\left(H_{n}^{2}+H_{n+1}^{2} i+H_{n+2}^{2} \varepsilon+H_{n+3}^{2} i \varepsilon\right) \\
& =\left(H_{n}^{1} \pm H_{n}^{2}\right)+\left(H_{n+1}^{1} \pm H_{n+1}^{2}\right) i+\left(H_{n+2}^{1} \pm H_{n+2}^{2}\right) \varepsilon+\left(H_{n+3}^{1} \pm H_{n+3}^{2}\right) i \varepsilon
\end{aligned}
$$

and

$$
\begin{aligned}
D C X_{n}^{1} \times D C X_{n}^{2} & =\left(R_{n}^{1}+R_{n}^{1 *} \varepsilon\right) \times\left(R_{n}^{2}+R_{n}^{2 *} \varepsilon\right) \\
& =\left(H_{n}^{1}+H_{n+1}^{1} i+H_{n+2}^{1} \varepsilon+H_{n+3}^{1} i \varepsilon\right) \times\left(H_{n}^{2}+H_{n+1}^{2} i+H_{n+2}^{2} \varepsilon+H_{n+3}^{2} i \varepsilon\right) \\
& =\left(H_{n}^{1} H_{n}^{2}-H_{n+1}^{1} H_{n+1}^{2}\right)+\left(H_{n}^{1} H_{n+1}^{2}+H_{n+1}^{1} H_{n}^{2}\right) i \\
& +\left(H_{n}^{1} H_{n+2}^{2}-H_{n+1}^{1} H_{n+3}^{2}-H_{n+3}^{1} H_{n+1}^{2}+H_{n+2}^{1} H_{n}^{2}\right) \varepsilon \\
& +\left(H_{n}^{1} H_{n+3}^{2}+H_{n+1}^{1} H_{n+2}^{2}+H_{n+2}^{1} H_{n+1}^{2}+H_{n+3}^{1} H_{n}^{2}\right) i \varepsilon,
\end{aligned}
$$

respectively. Since dual-complex numbers with generalized Fibonacci coefficients can be expressed as 


$$
D C X_{n}=R_{n}+R_{n}^{*} \varepsilon=\left(H_{n}+H_{n+1} i\right)+\left(H_{n+2}+H_{n+3} i\right) \varepsilon
$$

there exist five different conjugations. Then, we define these five different conjugates as

$$
\begin{array}{ll}
D C X_{n}^{\dagger_{1}}=\left(H_{n}-H_{n+1} i\right)+\left(H_{n+2}-H_{n+3} i\right) \varepsilon & \text { complex conjugation, } \\
D C X_{n}^{\dagger_{2}}=\left(H_{n}+H_{n+1} i\right)-\left(H_{n+2}+H_{n+3} i\right) \varepsilon & \text { dual conjugation, } \\
D C X_{n}^{\dagger_{3}}=\left(H_{n}-H_{n+1} i\right)-\left(H_{n+2}-H_{n+3} i\right) \varepsilon & \text { coupled conjugation, } \\
D C X_{n}^{\dagger_{4}}=\left(H_{n}-H_{n+1} i\right)\left(1-\frac{H_{n+2}+H_{n+3} i}{H_{n}+H_{n+1} i} \varepsilon\right) & \text { dual - complex conjugation, } \\
D C X_{n}^{\dagger_{5}}=\left(H_{n+2}+H_{n+3} i\right)-\left(H_{n}+H_{n+1} i\right) \varepsilon & \text { anti - dual conjugation. }
\end{array}
$$

By considering the definition of conjugates, five different norms for dual-complex numbers with generalized Fibonacci coefficients can be given as follows:

Definition 3.2. Let $D C X_{n}$ be a dual-complex number with generalized Fibonacci coefficient. Modulus of $D C X_{n}$ are denoted by $\left|D C X_{n}\right|_{\dagger_{i}}^{2}$ and $(i=1,2,3,4,5)$ are given by as follows

$$
\begin{aligned}
& \left|D C X_{n}\right|_{\dagger_{1}}^{2}=D C H_{n} \times D C H_{n}^{\dagger_{1}}, \\
& \left|D C X_{n}\right|_{\dagger_{2}}^{2}=D C H_{n} \times D C H_{n}^{\dagger_{2}}, \\
& \left|D C X_{n}\right|_{\dagger_{3}}^{2}=D C H_{n} \times D C H_{n}^{\dagger_{3}}, \\
& \left|D C X_{n}\right|_{\dagger_{4}}^{2}=D C H_{n} \times D C H_{n}^{\dagger_{4}}, \\
& \left|D C X_{n}\right|_{\dagger_{5}}^{2}=D C H_{n} \times D C H_{n}^{\dagger_{5}} .
\end{aligned}
$$

Proposition 3.1. Let us take the dual-complex number with generalized Fibonacci coefficient $D C X_{n} \in D C X$. Then, the following identities are satisfied:

$$
\begin{aligned}
& D C X_{n}+D C X_{n}^{\dagger_{1}}=2\left(H_{n}+H_{n+2} \varepsilon\right), \\
& D C X_{n} \times D C X_{n}^{\dagger_{1}}=(2 p-q)\left[H_{2 n+1}+2 H_{2 n+3} \varepsilon\right]-e\left[F_{2 n+1}+2 F_{2 n+3} \varepsilon\right], \\
& D C X_{n}+D C X_{n}^{\dagger_{2}}=2\left(H_{n}+H_{n+1} i\right), \\
& D C X_{n} \times D C X_{n}^{\dagger_{2}}=-H_{n-1} H_{n+2}+2 H_{n} H_{n+1} i, \\
& D C X_{n}+D C X_{n}^{\dagger_{3}}=2\left(H_{n}+H_{n+3} i \varepsilon\right), \\
& D C X_{n} \times D C X_{n}^{\dagger_{3}}=(2 p-q) H_{2 n+1}-e\left[F_{n+1}-2(-1)^{n} i \varepsilon\right] \\
& D C X_{n}+D C X_{n}^{\dagger_{4}}=2 H_{n}+\left(H_{n+2}-\frac{\left(H_{n}-H_{n+1} i\right)\left(H_{n+2}+H_{n+3} i\right)}{H_{n}+H_{n+1} i}\right) \varepsilon+H_{n+3} i \varepsilon, \\
& D C X_{n} \times D C X_{n}^{\dagger_{4}}=(2 p-q) H_{2 n+1}-e F_{2 n+1}, \\
& D C X_{n}+D C X_{n}^{\dagger_{5}}=H_{n}+H_{n+2}+\left(H_{n+1}+H_{n+3}\right) i+H_{n+1} \varepsilon+H_{n+2} i \varepsilon \\
& D C X_{n} \times D C X_{n}^{\dagger_{5}}=\left(H_{n} H_{n+2}-H_{n+1} H_{n+3}\right)+\left(H_{n} H_{n+3}+H_{n+1} H_{n+2}\right) i \\
& \quad+\left(H_{n-1} H_{n+2}-H_{n+1} H_{n+4}\right) \varepsilon+2\left(H_{n+2} H_{n+3}-H_{n} H_{n+1}\right) i \varepsilon .
\end{aligned}
$$


Proof. (3.11) From the equations (3.2), (3.4) and (3.5), we obtain

$$
D C X_{n}+D C X_{n}^{\dagger_{1}}=2\left(H_{n}+H_{n+2} \varepsilon\right) .
$$

Here, if $p=1$ and $q=0$ are specially taken for the generalized Fibonacci number $H_{n}$, then we reach $D C X_{n}+$ $D C X_{n}^{\dagger_{1}}=2\left(F_{n}+F_{n+2} \varepsilon\right)$.

(3.12) Considering the equations (3.3), (3.4), (3.5) and using the identities $H_{n-1}^{2}+H_{n}^{2}=(2 p-q) H_{2 n-1}-e F_{2 n-1}$ (see ref. [4]) and $H_{n} H_{m}+H_{n+1} H_{m+1}=(2 p-q) H_{n+m+1}-e F_{n+m+1}$ (see ref. [5]), we find

$$
\begin{aligned}
D C X_{n} \times D C X_{n}^{\dagger_{1}} & =\left(H_{n}^{2}+H_{n+1}^{2}\right)+2\left(H_{n} H_{n+2}+H_{n+1} H_{n+3}\right) \varepsilon \\
& =(2 p-q)\left[H_{2 n+1}+2 H_{2 n+3} \varepsilon\right]-e\left[F_{2 n+1}+2 F_{2 n+3} \varepsilon\right] .
\end{aligned}
$$

Here, if $p=1$ and $q=0$ are specially taken for the generalized Fibonacci number $H_{n}$, then we reach $D C X_{n} \times$ $D C X_{n}^{\dagger_{1}}=F_{2 n+1}+2 F_{2 n+3} \varepsilon$.

(3.13) From the equations (3.2), (3.4) and (3.6), we get

$$
D C X_{n}+D C X_{n}^{\dagger_{2}}=2\left(H_{n}+H_{n+1} i\right) .
$$

Here, if $p=1$ and $q=0$ are specially taken for the generalized Fibonacci number $H_{n}$, then we reach $D C X_{n}+$ $D C X_{n}^{\dagger_{1}}=2\left(F_{n}+F_{n+2} i\right)$.

(3.14) Using the equations (3.3), (3.4) and (3.6), we conclude that

$$
D C X_{n} \times D C X_{n}^{\dagger_{2}}=-H_{n-1} H_{n+2}+2 H_{n} H_{n+1} i .
$$

Here, if $p=1$ and $q=0$ are specially taken for the generalized Fibonacci number $H_{n}$, then we reach $D C X_{n} \times$ $D C X_{n}^{\dagger_{2}}=-F_{n-1} F_{n+2}+2 F_{n} F_{n+1} i$.

(3.15) From the equations (3.2), (3.4) and (3.7), the following equation is found

$$
D C X_{n}+D C X_{n}^{\dagger_{3}}=2\left(H_{n}+H_{n+3} i \varepsilon\right) .
$$

Here, if $p=1$ and $q=0$ are specially taken for the generalized Fibonacci number $H_{n}$, then we reach $D C X_{n}+$ $D C X_{n}^{\dagger_{3}}=2\left(F_{n}+F_{n+3} i \varepsilon\right)$.

(3.16) Using the equations (3.3), (3.4), (3.7) and the identities $H_{n-1}^{2}+H_{n}^{2}=(2 p-q) H_{2 n-1}-e F_{2 n-1}, H_{n} H_{n+r+1}-$ $H_{n-s} H_{n+r+s+1}=(-1)^{n+s} e F_{s} F_{r+s+1}$, (see ref. [4]), the multiplication of $D C X_{n}$ and $D C X_{n}^{\dagger}$ can be written as

$$
\begin{aligned}
D C X_{n} \times D C X_{n}^{\dagger_{3}} & =\left(H_{n}^{2}+H_{n+1}^{2}\right)+2\left(H_{n} H_{n+3}-H_{n+1} H_{n+2}\right) i \varepsilon \\
& =(2 p-q) H_{2 n+1}-e\left[F_{n+1}+2(-1)^{n} i \varepsilon\right] .
\end{aligned}
$$

Here, if $p=1$ and $q=0$ are specially taken for the generalized Fibonacci number $H_{n}$, then we reach $D C X_{n} \times$ $D C X_{n}^{\dagger_{3}}=F_{2 n+1}-2(-1)^{n} i \varepsilon$.

(3.17) From the equations (3.2), (3.4) and (3.8), we have

$$
D C X_{n}+D C X_{n}^{\dagger_{4}}=2 H_{n}+\left(H_{n+2}-\frac{\left(H_{n}-H_{n+1} i\right)\left(H_{n+2}+H_{n+3} i\right)}{H_{n}+H_{n+1} i}\right) \varepsilon+H_{n+3} i \varepsilon .
$$

Here, if $p=1$ and $q=0$ are specially taken for the generalized Fibonacci number $H_{n}$, then we reach $D C X_{n}+$ $D C X_{n}^{\dagger_{4}}=2 F_{n}+\left(F_{n+2}-\frac{\left(F_{n}-F_{n+1} i\right)\left(F_{n+2}+F_{n+3} i\right)}{F_{n}+F_{n+1} i}\right) \varepsilon+F_{n+3} i \varepsilon$.

(3.18) If we use the equations (3.3), (3.4), (3.8) and make the necessary calculations, then the rearranged equation (3.18) yields to

$$
D C X_{n} \times D C X_{n}^{\dagger}=(2 p-q) H_{2 n+1}-e F_{2 n+1} .
$$


Here, if $p=1$ and $q=0$ are specially taken for the generalized Fibonacci number $H_{n}$, then we reach $D C X_{n} \times$ $D C X_{n}^{\dagger_{4}}=F_{2 n+1}$.

(3.19) Considering the equations (3.2), (3.4) and (3.9), we find

$$
D C X_{n}+D C X_{n}^{\dagger_{5}}=H_{n}+H_{n+2}+\left(H_{n+1}+H_{n+3}\right) i+H_{n+1} \varepsilon+H_{n+2} i \varepsilon .
$$

Here, if $p=1$ and $q=0$ are specially taken for the generalized Fibonacci number $H_{n}$, then we reach $D C X_{n}+$ $D C X_{n}^{\dagger_{5}}=F_{n}+F_{n+2}+\left(F_{n+1}+F_{n+3}\right) i+F_{n+1} \varepsilon+F_{n+2} i \varepsilon$.

(3.20) From the equations (3.3), (3.4) and (3.9), the following identity is given by

$$
\begin{aligned}
D C X_{n} \times D C X_{n}^{\dagger_{5}} & =\left(H_{n} H_{n+2}-H_{n+1} H_{n+3}\right)+\left(H_{n} H_{n+3}+H_{n+1} H_{n+2}\right) i \\
& +\left(H_{n-1} H_{n+2}-H_{n+1} H_{n+4}\right) \varepsilon+2\left(H_{n+2} H_{n+3}-H_{n} H_{n+1}\right) i \varepsilon .
\end{aligned}
$$

Here, if $p=1$ and $q=0$ are specially taken for the generalized Fibonacci number $H_{n}$, then we reach

$$
\begin{aligned}
D C X_{n} \times D C X_{n}^{\dagger_{5}} & =\left(F_{n} F_{n+2}-F_{n+1} F_{n+3}\right)+\left(F_{n} F_{n+3}+F_{n+1} F_{n+2}\right) i \\
& +\left(F_{n-1} F_{n+2}-F_{n+1} F_{n+4}\right) \varepsilon+2\left(F_{n+2} F_{n+3}-F_{n} F_{n+1}\right) i \varepsilon .
\end{aligned}
$$

Theorem 3.1. Let $D C X_{n}$ and $D C X_{n-1}$ be two dual-complex Fibonacci numbers with generalized Fibonacci coefficients. The following identities are satisfied for these numbers and their conjugates.

i. $\left(D C X_{n} \times D C X_{n}^{\dagger_{1}}\right)+\left(D C X_{n-1} \times D C X_{n-1}^{\dagger_{1}}\right)=(2 p-q)\left[H_{2 n+1}+H_{2 n-1}+2\left(H_{2 n+3}+H_{2 n+1}\right) \varepsilon\right]$,

$$
-e\left[F_{2 n+1}+F_{2 n-1}+2\left(F_{2 n+3}+F_{2 n+1}\right) \varepsilon\right],
$$

ii. $D C X_{n}^{2}=2 H_{n} D C X_{n}-\left(D C X_{n} \times D C X_{n}^{\dagger_{1}}\right)+2 H_{n+2}\left(H_{n} \varepsilon+H_{n+1} i \varepsilon\right)$,

iii. $D C X_{n}^{2}+D C X_{n-1}^{2}=2(2 p-q) D C X_{2 n-1}-D C X_{n} \times D C X_{n}^{\dagger_{1}}-D C X_{n-1} \times D C X_{n-1}^{\dagger_{1}}$

$$
+(2 p-q)\left(H_{2 n-2}+4 H_{2 n+1}+2 H_{2 n+2} i \varepsilon\right)-e\left(F_{2 n+1}+2 F_{2 n} i-2\left(F_{2 n+2}+F_{2 n+4}\right) \varepsilon-4 F_{2 n+2} i \varepsilon\right),
$$

iv. $D C Y_{n} \times D C X_{n}^{\dagger_{1}}-D C Y_{n}^{\dagger_{1}} \times D C X_{n}=(-1)^{n}\left[\left(4 p^{2}-16 p q+8 q^{2}\right) i+\left(12 p^{2}\right) i \varepsilon\right]$.

Proof. i. Considering the equations (3.3), (3.4) and applying the identities $H_{n} H_{m}+H_{n+1} H_{m+1}=(2 p-q) H_{m+n+1}-$ $e F_{m+n+1}$ (see ref. [5]), $H_{n-1}^{2}+H_{n}^{2}=(2 p-q) H_{2 n-1}-e F_{2 n-1}$ (see ref. [4]), the proof is completed.

ii. Taking into account the equality (3.2), the proof can be easily seen.

iii. If we use the identity $H_{n} H_{m}+H_{n+1} H_{m+1}=(2 p-q) H_{m+n+1}-e F_{m+n+1}$ (see ref. [5]) and equation (3.2), the proof is completed.

iv. The desired result can be obtained by the help of the equation (3.2) and the identity $L_{n} F_{n}=F_{m+n}+(-1)^{m} F_{m-n}$ (see ref [4]).

Also, the equations given in the Proposition 2.2 which have been handled in the article [2] are obtained by giving the special values $p=1$ and $q=0$ in the above equations.

i. $\left(D C X_{n} \times D C X_{n}^{\dagger_{1}}\right)+\left(D C X_{n-1} \times D C X_{n-1}^{\dagger_{1}}\right)=F_{2 n+1}+F_{2 n-1}+2\left(F_{2 n+3}+F_{2 n+1}\right) \varepsilon$,

ii. $D C X_{n}^{2}=2 F_{n} D C F_{n}-D C F_{n} \times D C F_{n}^{\dagger_{1}}+2 F_{n+2}\left(F_{n} \varepsilon+F_{n+1} i \varepsilon\right)$,

iii. $D C X_{n}^{2}+D C X_{n-1}^{2}=4 D C F_{2 n-1}-D C F_{n} \times D C F_{n}^{\dagger_{1}}-D C F_{n-1} \times D C F_{n-1}^{\dagger_{1}}$

$$
+\left(2 F_{2 n-2}+7 F_{2 n+1}-2 F_{2 n} i+2\left(F_{2 n+2}+F_{2 n+4}\right) \varepsilon+8 F_{2 n+2} i \varepsilon\right) \text {, }
$$

iv. $D C Y_{n} \times D C X_{n}^{\dagger_{1}}-D C Y_{n}^{\dagger_{1}} \times D C X_{n}=(-1)^{n}[4 i+12 i \varepsilon]$.

Theorem 3.2. Let $D C X_{n}$ be dual-complex number with generalized Fibonacci coefficient. In this case, for $n \geq 0$, the following identities are given: 
i. $D C X_{n}+D C X_{n+1}=D C X_{n+2}$.

ii. $\left(D C X_{n}\right)^{2}=2\left(H_{n} D C X_{n}\right)-2\left(H_{n+1} D C X_{n+1}\right)-H_{n}+2\left(H_{n+4}+H_{n+1} H_{n+2}\right) i \varepsilon$.

iii. $D C X_{n}-D C X_{n+1} i-D C X_{n+2} \varepsilon+D C X_{n+3} i \varepsilon=-H_{\mathrm{n}+1}$.

iv. $\left(D C X_{n} \times D C X_{m}\right)+\left(D C X_{n+1} \times D C X_{m+1}\right)=D C X_{m+n+1}+\left[-(2 p-q) H_{m+n+2}-H_{m+n+1}+e F_{m+n+2}\right]$

$$
\begin{aligned}
& +i\left[2(2 p-q) H_{m+n+2}-H_{m+n+2}-2 e F_{m+n+2}\right] \\
& +\varepsilon\left[-2(2 p-q) H_{m+n+4}-H_{m+n+3}+2 e F_{m+n+4}\right] \\
& +i \varepsilon\left[4(2 p-q) H_{m+n+4}-H_{m+n+4}-4 e F_{m+n+4}\right] .
\end{aligned}
$$

v. $D C X_{n}^{2}+D C X_{n-1}^{2}=2\left[(2 p-q)\left(H_{2 n} i-H_{2 n+2} \varepsilon+2 H_{2 n+2} i \varepsilon\right)\right]-2 e\left[F_{2 n} i-F_{2 n+2} \varepsilon+2 F_{2 n+2} i \varepsilon\right]$.

Proof. i. Let $D C H_{n}$ and $D C H_{n+1}$ be two dual-complex numbers with generalized Fibonacci coefficients. If we take into account the equation (3.2), then the following identity can be easily seen

$$
D C H_{n}+D C H_{n+1}=H_{n+2}+H_{n+3} i+H_{n+4} \varepsilon+H_{n+5} i \varepsilon=D C H_{n+2} .
$$

Specially, if the values $p=1$ and $q=0$ are taken in the generalized Fibonacci number $H_{n}$, then the identity

$$
D C X_{n}+D C X_{n+1}=D C F_{n+2}
$$

is obtained.

ii. Let $D C H_{n}$ be a dual-complex number with generalized Fibonacci coefficients. In this case, the equation (3.3) gives us

$$
\begin{aligned}
D C X_{n}^{2} & =\left[\left(H_{n}+H_{n+1} i\right)+\left(H_{n+2}+H_{n+3} i\right) \varepsilon\right] \times\left[\left(H_{n}+H_{n+1} i\right)+\left(H_{n+2}+H_{n+3} i\right) \varepsilon\right] \\
& =2\left(H_{n} D C X_{n}\right)-2\left(H_{n+1} D C X_{n+1}\right)-H_{n}+2\left(H_{n+4}+H_{n+1} H_{n+2}\right) i \varepsilon+2 H_{n+1}\left(H_{n+4}+H_{n+2}\right) i \varepsilon .
\end{aligned}
$$

Specially, if the values $p=1$ and $q=0$ are taken in the generalized Fibonacci number $H_{n}$, then the identity

$$
\left(D C X_{n}\right)^{2}=2\left(F_{n} D C F_{n}\right)-2\left(F_{n+1} D C F_{n+1}\right)-F_{n}+2\left(F_{n+4}+F_{n+1} F_{n+2}\right) i \varepsilon
$$

is found.

iii. By using the equation (3.2) and making the direct calculations, we get

$$
\begin{aligned}
D C X_{n}-D C X_{n+1} i-D C X_{n+2} \varepsilon+D C X_{n+3} i \varepsilon & =\left[\left(H_{n}+H_{n+1} i\right)+\left(H_{n+2}+H_{n+3} i\right) \varepsilon\right] \\
& -\left[\left(H_{n+1}+H_{n+2} i\right)+\left(H_{n+3}+H_{n+4} i\right) \varepsilon\right] i \\
& -\left[\left(H_{n+2}+H_{n+3} i\right)+\left(H_{n+4}+H_{n+5} i\right) \varepsilon\right] \varepsilon \\
& +\left[\left(H_{n+3}+H_{n+4} i\right)+\left(H_{n+5}+H_{n+6} i\right) \varepsilon\right] i \varepsilon \\
& =-H_{n+1 .}
\end{aligned}
$$

Specially, if we substitute the values $p=1$ and $q=0$ into the generalized Fibonacci number $H_{n}$, then we have

$$
D C X_{n}-i D C X_{n+1}-\varepsilon D C X_{n+2}+i \varepsilon D C X_{n+3}=-F_{\mathrm{n}+1} .
$$

iv. From the equation (3.3) $D C X_{n} \times D C X_{m}+D C X_{n+1} \times D C X_{m+1}=\left[H_{n}+H_{n+1} i+\left(H_{n+2}+H_{n+3} i\right) \varepsilon\right] \times\left[H_{m}+H_{m+1} i+\left(H_{m+2}+H_{m+3} i\right) \varepsilon\right]$
$+\left[H_{n+1}+H_{n+2} i+\left(H_{n+3}+H_{n+4} i\right) \varepsilon\right] \times\left[H_{m+1}+H_{m+2} i+\left(H_{m+3}+H_{m+4} i\right) \varepsilon\right]$ 
can be written. In order to simplify further algebraic manipulations we shall use $H_{n} H_{m}+H_{n+1} H_{m+1}=(2 p-q) H_{m+n+1}$ $-e F_{m+n+1}$ (see ref. [5]). Thus, we have

$$
\begin{aligned}
\left(D C X_{n} \times D C X_{m}\right)+\left(D C X_{n+1} \times D C X_{m+1}\right) & =D C X_{m+n+1}+\left[-(2 p-q) H_{m+n+2}-H_{m+n+1}+e F_{m+n+2}\right] \\
& +i\left[2(2 p-q) H_{m+n+2}-H_{m+n+2}-2 e F_{m+n+2}\right] \\
& +\varepsilon\left[-2(2 p-q) H_{m+n+4}-H_{m+n+3}+2 e F_{m+n+4}\right] \\
& +i \varepsilon\left[4(2 p-q) H_{m+n+4}-H_{m+n+4}-4 e F_{m+n+4}\right] .
\end{aligned}
$$

Specially, if we substitute the values $p=1$ and $q=0$ into the generalized Fibonacci number $H_{n}$, then the following identity is found

$$
\left(D C X_{n} \times D C X_{m}\right)+\left(D C X_{n+1} \times D C X_{m+1}\right)=D C F_{n+m+1}-F_{n+m+3}+F_{n+m+2} i-F_{n+m+6} \varepsilon+3 F_{n+m+4} i \varepsilon .
$$

v. The desired result is reached from identity $H_{n} H_{m}+H_{n+1} H_{m+1}=(2 p-q) H_{m+n+1}-e F_{m+n+1}$ (see ref. [5]) and the equation (3.3).

Specially, if we substitute the values $p=1$ and $q=0$ into the generalized Fibonacci number $H_{n}$, then the following identity is obtained

$$
D C X_{n}^{2}+D C X_{n-1}^{2}=2\left[i F_{2 n}-\varepsilon F_{2 n+2}+2 i \varepsilon F_{2 n+2}\right] .
$$

Theorem 3.3. Let $D C X_{n}$ and $D C L_{n}$ be dual-complex numbers with generalized Fibonacci and Lucas coefficients, respectively. For $n \geq 0$, the following relations can be given between these numbers

i. $D C X_{n+1}+D C X_{n-1}=p D C L_{n}+q D C L_{n}$

and

ii. $D C X_{n+2}-D C X_{n-2}=p D C L_{n}+q D C L_{n}$.

Proof. Let us consider the identity $H_{n+1}+H_{n-1}=p L_{n}+q L_{n-1}$ (see ref. [11]) and the recurrence relation $H_{n}=$ $(p-q) F_{n}+q F_{n+1}$ to prove i. and ii., respectively. Thus, we have

$$
\begin{aligned}
D C X_{n+1}+D C X_{n-1} & =\left(H_{n+1}+H_{n+2} i+H_{n+3} \varepsilon+H_{n+4} i \varepsilon\right) \\
& +\left(H_{n-1}+H_{n} i+H_{n+1} \varepsilon+H_{n+3} i \varepsilon\right) \\
& =\left(H_{n+1}+H_{n-1}\right)+\left(H_{n+2}+H_{n}\right) i+\left(H_{n+3}+H_{n+1}\right) \varepsilon \\
& +\left(H_{n+4}+H_{n+3}\right) i \varepsilon \\
& =\left(p L_{n}+q L_{n-1}\right)+\left(p L_{n-1}+q L_{n}\right) i+\left(p L_{n+2}+q L_{n+1}\right) \varepsilon \\
& +\left(q L_{n+3}+q L_{n+2}\right) i \varepsilon \\
& =p D C L_{n}+q D C L_{n}
\end{aligned}
$$

and

$$
\begin{aligned}
D C X_{n+2}-D C X_{n-2} & =\left(H_{n+2}+H_{n+3} i+H_{n+4} \varepsilon+H_{n+5} i \varepsilon\right) \\
& -\left(H_{n-2}+H_{n-1} i+H_{n} \varepsilon+H_{n+1} i \varepsilon\right) \\
& =\left(H_{n+2}-H_{n-2}\right)+\left(H_{n+3}-H_{n-1}\right) i+\left(H_{n+4}-H_{n}\right) \varepsilon \\
& +\left(H_{n+5}-H_{n+1}\right) i \varepsilon \\
& =\left(p L_{n}+q L_{n-1}\right)+\left(p L_{n-1}+q L_{n}\right) i+\left(p L_{n+2}+q L_{n+1}\right) \varepsilon \\
& +\left(q L_{n+3}+q L_{n+2}\right) i \varepsilon \\
& =p D C L_{n}+q D C L_{n} .
\end{aligned}
$$

Specially, if we substitute the values $p=1$ and $q=0$ into the generalized Fibonacci number $H_{n}$, then we get $D C X_{n+1}+D C X_{n-1}=D C L_{n}$ and $D C X_{n+2}-D C X_{n-2}=D C L_{n}$.

Theorem 3.4. The sums of the dual-complex numbers with generalized Fibonacci coefficients satisfy the following relations:

i. $\sum_{s=1}^{n} D C X_{s}=D C X_{n+2}-D C X_{2}$, 
ii. $\sum_{s=0}^{p} D C X_{s+n}+D C X_{x+1}=D C X_{n+p+2}$,

iii. $\sum_{s=1}^{n} D C X_{2 s-1}=D C X_{2 n}-D C X_{0}$,

iv. $\sum_{s=1}^{n} D C X_{2 s}=D C X_{2 n+1}-D C X_{1}$.

Proof. By using the identity $\sum_{t=a}^{n} H_{t}=H_{n+2}-H_{a+1}$ (see ref. [11]), it can be easily seen that

i. $\sum_{s=1}^{n} D C X_{s}=\sum_{s=1}^{n} H_{s}+i \sum_{s=1}^{n} H_{s+1}+\varepsilon \sum_{s=1}^{n} H_{s+2}+i \varepsilon \sum_{s=1}^{n} H_{s+3}=D C X_{n+2}-D C X_{2}$.

Proofs of ii, iii, iv are done in a similar manner.

Specially, if we substitute the values $p=1$ and $q=0$ into the generalized Fibonacci number $H_{n}$, then the following identities are found

i. $\sum_{s=1}^{n} D C X_{s}=D C F_{n+2}-D C F_{2}$,

ii. $\sum_{s=0}^{p} D C X_{s+n}+D C X_{x+1}=D C F_{n+p+2}$,

iii. $\sum_{s=1}^{n} D C X_{2 s-1}=D C F_{2 n}-D C F_{0}$,

iv. $\sum_{s=1}^{n} D C X_{2 s}=D C F_{2 n+1}-D C F_{1}$.

Now, let's find the correspondence of Binet formula for the dual-complex Fibonacci numbers, which helps to obtain the golden ratio (which allows to find the $n$-th term of dual-complex numbers with generalized Fibonacci coefficients sequence).

Theorem 3.5 (Binet's Formula). Let $D C X_{n}$ be the dual-complex number with generalized Fibonacci coefficients. For $m, n \geq 1$, Binet's formula is as follows:

$$
D C X_{n}=\frac{\bar{\alpha} \alpha^{n}-\bar{\beta} \beta^{n}}{\alpha-\beta}
$$

where

$$
\bar{\alpha}=(p-q \beta)+[p(1-\beta)+q] i+[p(2-\beta)+q(1-\beta)] \varepsilon+[p(3-2 \beta)+q(2-\beta)] i \varepsilon
$$

and

$$
\bar{\beta}=(p-q \alpha)+[p(1-\alpha)+q] i+[p(2-\alpha)+q(1-\alpha)] \varepsilon+[p(3-2 \alpha)+q(2-\alpha)] i \varepsilon,
$$

such that $\alpha$ and $\beta$ are the roots of the equation $t^{2}-t-1=0$.

Proof. If $t_{1}$ and $t_{2}$ denote the roots of characteristic equation $t^{2}-t-1=0$ associated to the recurrence relation $D C X_{n}+D C X_{n+1}=D C X_{n+2}$. Then, these roots are be found as $\alpha=t_{1}=\frac{1+\sqrt{5}}{2}$ and $\beta=t_{2}=\frac{1-\sqrt{5}}{2}$. Note that, $\alpha+\beta=1, \quad \alpha \cdot \beta=-1$ and $\alpha-\beta=\sqrt{5}$. Therefore, the general term of the dual-complex number sequence with generalized Fibonacci coefficients may be expressed in the form:

$$
D C X_{n}=A \alpha^{n}+B \beta^{n}
$$

for some coefficients $A$ and $B$. For $n=0$ and $n=1$, the following equalities can be written

$$
D C X_{0}=(q, \quad p, \quad p+q, 2 p+q)
$$

and

$$
D C X_{1}=(p, p+q, 2 p+q, 3 p+2 q) .
$$

Also, if we consider the values $n=0$ and $n=1$, we get

$$
D C X_{0}=A+B
$$


and

$$
D C X_{1}=\alpha A+\beta B .
$$

Then, solving these system of linear equations, we have

$$
A=\frac{D C X_{1}-\beta D C X_{0}}{\alpha-\beta} \text { and } B=\frac{\alpha D C X_{0}-D C X_{1}}{\alpha-\beta},
$$

where the coefficients $\bar{\alpha}$ and $\bar{\beta}$ are

$$
\bar{\alpha}=(p-q \beta)+[p(1-\beta)+q] i+[p(2-\beta)+q(1-\beta)] \varepsilon+[p(3-2 \beta)+q(2-\beta)] i \varepsilon
$$

and

$$
\bar{\beta}=(p-q \alpha)+[p(1-\alpha)+q] i+[p(2-\alpha)+q(1-\alpha)] \varepsilon+[p(3-2 \alpha)+q(2-\alpha)] i \varepsilon .
$$

Now, let's find the generating function for the dual-complex numbers with generalized coefficients which allows us to obtain the Binet formula.

Theorem 3.6 (Generating Function). Generating function for dual-complex numbers with generalized Fibonacci coefficients is given by

$$
g(x)=\frac{1}{1-x-x^{2}} \sum_{s=0}^{3}\left(D C X_{s}+D C X_{s-1} x\right) e_{s} .
$$

Proof. The ordinary generating function associated with dual-complex numbers with generalized Fibonacci coefficients is defined by

$$
g(x)=\sum_{n=0}^{\infty} P_{n} x^{n}
$$

where $P_{n}=\left(D C X_{n}, D C X_{n+1}, D C X_{n+2}, D C X_{n+3}\right)$. Multiplying by $x$ and $x^{2}$ on both sides of the above equation respectively, we get

$$
\begin{aligned}
& x g(x)=P_{0} x+P_{1} x^{2}+\ldots+P_{n-1} x^{n}+\ldots \\
& x^{2} g(x)=P_{0} x^{2}+P_{1} x^{3}+\ldots+P_{n-2} x^{n}+\ldots .
\end{aligned}
$$

Substracting the above equations from $g(x)$ and rearranging, we obtain

$$
\left(1-x-x^{2}\right) g(x)=\sum_{s=0}^{3}\left(P_{0}+\left(P_{1}-P_{0}\right) x\right) .
$$

Finally, the generating function of dual-complex numbers with generalized Fibonacci coefficients can be written as

$$
g(x)=\frac{1}{1-x-x^{2}} \sum_{s=0}^{3}\left(P_{0}+\left(P_{1}-P_{0}\right) x\right) .
$$

Thus, the proof is completed.

Now, let's write the Binet formula in terms of the generating function by using the generating function which has been obtained in Theorem 3.6.

Theorem 3.7. Binet formula for the dual-complex numbers with generalized Fibonacci coefficients is

$$
P_{n}=P_{1} H_{n}+P_{0} H_{n-1} .
$$

Proof. Since the equation $t^{2}-t-1=0$ has two distinct roots which are denoted by $\alpha$ and $\beta$, then the sequence

$$
P_{n}=A \alpha^{n}+B \beta^{n}
$$

is the solution of the equation recurrence relation $D C X_{n}+D C X_{n+1}=D C X_{n+2}$. For the values $n=0$ and $n=1$, if we take

$$
A=\frac{P_{1}-\beta P_{0}}{\alpha-\beta} \quad \text { and } \quad B=\frac{\alpha P_{0}-P_{1}}{\alpha-\beta}
$$


therefore, the following equation can be written

$$
P_{n}=\frac{1}{\alpha-\beta}\left[\left(P_{1}-\beta P_{0}\right) \alpha^{n}+\left(\alpha P_{0}-P_{1}\right) \beta^{n}\right] .
$$

Then, writing the correspondences of $P_{0}$ and $P_{1}$ which have been obtained in Theorem 3.6 and rearranging the equation

$$
P_{n}=\left(\frac{\alpha^{n}-\beta^{n}}{\alpha-\beta}\right) \sum_{s=0}^{3} D C X_{s+1} e_{s}+\left(\frac{\alpha^{n-1}-\beta^{n-1}}{\alpha-\beta}\right) \sum_{s=0}^{3} D C X_{s} e_{s}
$$

is found.

Finally, we have

$$
P_{n}=P_{1} H_{n}+P_{0} H_{n-1} .
$$

Let's express the Catalan identity which is well-known identity for Fibonacci numbers.

Theorem 3.8 (Catalan's Identity). For $r \leq n$, Catalan identity the dual-complex numbers with generalized Fibonacci coefficients is

$$
D C X_{n}^{2}-D C X_{n+r} \times D C X_{n-r}=(-1)^{n-r} F_{r}\left[2 e F_{r}+\mu F_{r} i+3 F_{r}(2 \varepsilon+i \varepsilon) \mu\right] .
$$

Proof. From the identity $H_{m+k} H_{n-k}-H_{m} H_{n}=(-1)^{n-k+1} \mu F_{k} F_{m+k-n}$ (see ref. [6]), one can write

$$
D C X_{n}^{2}=H_{n}^{2}-H_{n+1}^{2}+2 H_{n} H_{n+1} i+2\left(H_{n} H_{n+2}-H_{n+1} H_{n+3}\right) \varepsilon+2\left(H_{n+1} H_{n+2}+H_{n} H_{n+3}\right) i \varepsilon
$$

and

$$
\begin{aligned}
D C X_{n+r} \times D C X_{n-r} & =H_{n+r} H_{n-r}-H_{n+r+1} H_{n-r+1}+\left(H_{n+r+1} H_{n-r}+H_{n+r} H_{n-r+1}\right) i \\
& +\left(H_{n+r} H_{n-r+2}-H_{n+r+1} H_{n-r+3}+H_{n-r+2} H_{n-r}-H_{n-r+3} H_{n-r+1}\right) \varepsilon \\
& +\left(H_{n+r+1} H_{n-r+2}+H_{n+r} H_{n-r+3}+H_{n+r+3} H_{n-r}+H_{n+r+2} H_{n-r+1}\right) i \varepsilon .
\end{aligned}
$$

The proof is completed by addition of the two equations. If the values $p=1$ and $q=0$ is taken into the Catalan identity which has been obtained for generalized dual-complex numbers with Fibonacci coefficients, then we find the Catalan identity for dual-complex numbers. Namely,

$$
D C X_{n}^{2}-D C X_{n+r} \times D C X_{n-r}=(-1)^{n-r} F_{r}^{2}(2+i+6 \varepsilon+3 i \varepsilon) .
$$

Let's give the identity of Cassini for generalized dual-complex numbers which is a special form of Catalan identity.

Theorem 3.9 (Cassini's Identity). For $1 \leq n$, Cassini identity for the dual-complex numbers with generalized Fibonacci coefficients is

$$
D C X_{n}^{2}-\left(D C X_{n+1} \times D C X_{n-1}\right)=(-1)^{n-1} e(2+i+6 \varepsilon+3 i \varepsilon) .
$$

Proof. In the particular case when $r=1$, Catalan identity which has been proved in Theorem 3.8 turns into Cassini identity for the dual-complex numbers with generalized Fibonacci coefficients.

If the values $p=1$ and $q=0$ is taken into the Cassini identity which is obtained for generalized dual-complex numbers with Fibonacci coefficients, then we find the Cassini identity for dual-complex numbers.

Theorem 3.10 (Honsberger Identity). For $0 \leq k, n$, Honsberger Identity for the dual-complex numbers with generalized Fibonacci coefficients $D C X_{n}$ and $D C X_{k}$ is given by

$$
\begin{aligned}
\left(D C X_{k-1} \times D C X_{n}\right)+\left(D C X_{k} \times D C X_{n+1}\right) & =-\left[(2 p-q) H_{k+n+1}-e F_{k+n+1}\right] \\
& +2 i\left[(2 p-q) H_{k+n+1}-e F_{k+n+1}\right] \\
& -2 \varepsilon\left[(2 p-q) H_{k+n+3}-e F_{k+n+3}\right] \\
& +4 i \varepsilon\left[(2 p-q) H_{k+n+3}-e F_{k+n+3}\right] .
\end{aligned}
$$


Proof. By using identity $H_{n} H_{m}+H_{n+1} H_{m+1}=(2 p-q) H_{m+n+1}-e F_{m+n+1}$ (see ref. [5]) and equations (3.2), (3.3) the proof can be easily seen.

Specially, if we substitute the values $p=1$ and $q=0$ into the generalized Fibonacci number $H_{n}$, then the following identity is obtained

$$
\left(D C X_{k-1} \times D C X_{n}\right)+\left(D C X_{k} \times D C X_{n+1}\right)=-2\left(F_{k+n+1}\right)+4 i\left(F_{k+n+1}\right)-4 \varepsilon\left(F_{k+n+3}\right)+8 i \varepsilon\left(F_{k+n+3}\right) .
$$

Theorem 3.11 (Tagiuri Identity). Between the dual-complex numbers with generalized Fibonacci coefficients and the Fibonacci numbers it is verified

$$
\left(D C X_{m+k} \times D C X_{n-k}\right)-\left(D C X_{m} \times D C X_{n}\right)=\mu F_{k}(-1)^{n-k+1} F_{m+k-n}(2+i+6 \varepsilon+3 i \varepsilon) .
$$

Proof. From the identity $H_{m+k} H_{n-k}-H_{m} H_{n}=(-1)^{n-k+1} \mu F_{k} F_{m+k-n}$ (see ref. [6]) and equations (3.2), (3.3) the proof can be clearly seen.

Specially, if we substitute the values $p=1$ and $q=0$ into the generalized Fibonacci number $H_{n}$, then this yields to

$$
\left(D C X_{m+k} \times D C X_{n-k}\right)-\left(D C X_{m} \times D C X_{n}\right)=F_{k}(-1)^{n-k+1} F_{m+k-n}(2+i+6 \varepsilon+3 i \varepsilon) .
$$

Theorem 3.12 (d'Ocagne's Identity). Let $D C X_{n}$ be a dual-complex number with generalized Fibonacci coefficients. If $n$ is a non-negative integer number and $m$ any natural number for $m>n$, the relation

$$
\left(D C X_{m} \times D C X_{n+1}\right)-\left(D C X_{n} \times D C X_{m+1}\right)=\mu F_{m-n}(-1)^{n}(2+i+6 \varepsilon+3 i \varepsilon)
$$

is verified.

Proof. If we take into consider the equations (3.2), (3.3) and use the identity $H_{m+k} H_{n-k}-H_{m} H_{n}=(-1)^{n-k+1} \mu F_{k} F_{m+k-n}$ (see ref. [6]), we complete the proof. Specially, if we substitute the values $p=1$ and $q=0$ into the generalized Fibonacci number $H_{n}$, then the d'Ocagne's Identity which is found for the dual-complex numbers with generalized Fibonacci coefficients becomes

$$
\left(D C X_{m} \times D C X_{n+1}\right)-\left(D C X_{n} \times D C X_{m+1}\right)=F_{m-n}(-1)^{n}(2+i+6 \varepsilon+3 i \varepsilon) .
$$

\section{Conclusions}

This study fills a gap regarding dual-complex numbers with generalized Fibonacci and Lucas coefficients in the literature by using the definitions of dual-complex Fibonacci and Lucas numbers. For this purpose, the generating function of dual-complex numbers with generalized Fibonacci coefficients have been firstly found. Then, Binet's formula has been given with the help of this generating function. Binet's formula has been also proved in a second way by using the roots of the characteristic equation. However, Cassini, Catalan, d'Ocagne's, Honsberger, Tagiuri and other identities have been proved for this new number system and it is seen that these identities correspond to the theorems in the article [2] for the values $p=1$ and $q=0$. In addition, it is seen that dual-complex number system with generalized Fibonacci and Lucas coefficient has an algebraic structure according to the multiplication process. Hence, there are five different conjugates for these numbers. Thus, in addition to the identities related to the conjugates which has been obtained in Theorem 3.1, the following identities are given:

$$
\begin{aligned}
\left(D C X_{n} \times D C X_{n}^{\dagger_{2}}\right)+\left(D C X_{n-1} \times D C X_{n-1}^{\dagger_{2}}\right) & =(-1+2 i)\left[(2 p-q) H_{2 n}-e F_{2 n}\right], \\
\left.D C X_{n} \times D C X_{n}^{\dagger_{3}}\right)+\left(D C X_{n-1} \times D C X_{n-1}^{\dagger_{3}}\right) & =(2 p-q)\left[H_{2 n-1}+H_{2 n+1}\right]-e\left[F_{2 n-1}+F_{2 n+1}\right], \\
\left(D C X_{n} \times D C X_{n}^{\dagger_{4}}\right)+\left(D C X_{n-1} \times D C X_{n-1}^{\dagger_{4}}\right) & =(2 p-q)\left[H_{2 n+1}+H_{2 n-1}\right]-e\left[F_{2 n+1}+F_{2 n-1}\right], \\
\left.D C X_{n} \times D C X_{n}^{\dagger_{5}}\right)+\left(D C X_{n-1} \times D C X_{n-1}^{\dagger_{5}}\right) & =(2 p-q)\left[H_{2 n+2}(1+2 i)+H_{2 n+1}(-\varepsilon+2 i \varepsilon)\right] \\
& -e\left[F_{2 n+2}(1+2 i)+F_{2 n+1}(-\varepsilon+2 i \varepsilon)\right],
\end{aligned}
$$




$$
\begin{aligned}
& D C X_{n}^{2}=2 H_{n} D C X_{n}-D C X_{n} \times D C X_{n}^{\dagger 2}+2 H_{n+1}\left(-H_{n+1}+H_{n} i-H_{n+3} \varepsilon+H_{n+2} i \varepsilon\right), \\
& D C X_{n}^{2}=2 H_{n} D C X_{n}-D C X_{n} \times D C X_{n}^{\dagger 3}+2 H_{n+3}\left(-H_{n+1} \varepsilon+H_{n} i \varepsilon\right) \text {, } \\
& D C X_{n}^{2}=2 H_{n} D C X_{n}-D C X_{n} \times D C X_{n}^{\dagger}{ }^{4}+2 H_{n+1}\left(-H_{n+3} \varepsilon+H_{n+2} i \varepsilon\right) \text {, } \\
& D C X_{n}^{2}=2 H_{n} D C X_{n}-D C X_{n} \times D C X_{n}^{\dagger 5}+\left(H_{n} H_{n+2}-H_{n+1} H_{n+3}-H_{n}^{2}-H_{n+1}^{2}\right)+\left(H_{n+1} H_{n+2}+H_{n} H_{n+3}\right) i \\
& +\left(H_{n-1} H_{n+2}-H_{n+1} H_{n+3}-H_{n+1} H_{n+5}\right) \varepsilon+2\left(H_{n+3} H_{n+2}+H_{n+1}^{2}\right) i \varepsilon \\
& D C X_{n}^{2}+D C X_{n-1}^{2}=2(2 p-q) D C X_{2 n-1}-D C X_{n} \times D C X_{n}^{\dagger_{2}}-D C X_{n-1} \times D C X_{n-1}^{\dagger_{2}} \\
& +(2 p-q)\left(-2 H_{2 n+1}+2 H_{2 n} i-2 H_{2 n+2} \varepsilon+2 H_{2 n+2} i \varepsilon\right) \\
& -e\left(-2 F_{2 n}+4 F_{2 n} i-2\left(F_{2 n+2}+F_{2 n}\right) \varepsilon+4 F_{2 n+2} i \varepsilon\right) \text {, } \\
& D C X_{n}^{2}+D C X_{n-1}^{2}=2(2 p-q) D C X_{2 n-1}-D C X_{n} \times D C X_{n}^{\dagger_{3}}-D C X_{n-1} \times D C X_{n-1}^{\dagger_{3}} \\
& +(2 p-q)\left(-2 H_{2 n+2} \varepsilon+2 H_{2 n+2} i \varepsilon\right) \\
& -e\left(F_{2 n-1}+2 F_{2 n} i-2\left(F_{2 n+2}+F_{2 n}\right) \varepsilon+4 F_{2 n+2} i \varepsilon\right) \text {, } \\
& D C X_{n}^{2}+D C X_{n-1}^{2}=2(2 p-q) D C X_{2 n-1}-D C X_{n} \times D C X_{n}^{\dagger_{4}}-D C X_{n-1} \times D C X_{n-1}^{\dagger_{4}} \\
& +(2 p-q)\left(-2 H_{2 n+2} \varepsilon+2 H_{2 n+2} i \varepsilon\right) \\
& -e\left(F_{2 n-1}+2 F_{2 n} i-2\left(F_{2 n+2}+F_{2 n}\right) \varepsilon+4 F_{2 n+2} i \varepsilon\right) \text {, } \\
& D C X_{n}^{2}+D C X_{n-1}^{2}=2(2 p-q) D C X_{2 n-1}-D C X_{n} \times D C X_{n}^{\dagger_{5}}-D C X_{n-1} \times D C X_{n-1}^{\dagger_{5}} \\
& +(2 p-q)\left(H_{2 n-2}+2 H_{2 n+2} i-H_{2 n+4} \varepsilon+2 H_{2 n+3} i \varepsilon\right) \\
& -e\left(F_{2 n+1}+2\left(F_{2 n+2}+F_{2 n}\right) i+\left(2\left(F_{2 n-1}-F_{2 n+3}\right)-F_{2 n+1}\right) \varepsilon+6 F_{2 n+2} i \varepsilon\right) \text {. }
\end{aligned}
$$

\section{References}

[1] Dunlap, A.R.: The golden ratio and Fibonacci numbers. World Scientific Publishing Co. Pte. Ltd. Singapore (1997).

[2] Güngör, M.A., Azak, A.Z.: Investigation of dual-complex Fibonacci, dual-complex Lucas numbers and their properties. Advances in Applied Clifford Algebras. 27 (4), 3083-3096 (2017).

[3] Hoggatt, Jr.V.E.: Fibonacci and Lucas Numbers. Mifflin Co. Boston (1969).

[4] Horadam, A.F.: A generalized Fibonacci sequence. The American Mathematical Monthly. 68 (5), 455-459 (1961).

[5] Iakini, A.L.: Generalized quaternions of higher order. The Fibonacci Quarterly. 15 (4), 343-346 (1977).

[6] Koshy, T.: Fibonacci and Lucas numbers with applications. Wiley and Sons Publication New York (2001).

[7] Majernik, V.: Multicomponent number systems. Acta Physica Polonica Series a. 90 (3), 491-498 (1996).

[8] Messelmi, F.: Dual-complex numbers and their holomorphic functions.https://hal.archives-ouvertes.fr/hal01114178, (2015).

[9] Scholfield, P.H.: The Theory of Proportion in Architecture. Cambridge University Press Cambridge (1958).

[10] Silvester, J.R.: Fibonacci properties by matrix methods. The Mathematical Gazette. 63 (425), 188-191 (1979).

[11] Yüce, S., Aydın Torunbalcı, F.: Generalized Dual Fibonacci Quaternions. Applied Mathematics E-Notes. 16 (30), 276-289 (2016).

\section{Affiliations}

ARZU CIHAN

ADDRESS: Sakarya University, Dept. of Mathematics, 54187, Sakarya-TURKEY.

E-MAIL: arzu.cihan3@ogr.sakarya.edu.tr

ORCID ID:0000-0003-2003-3507

AYŞE ZEYNEP AZAK

ADDRESS: Sakarya University, Dept. of Mathematics and Science Education, 54300, Sakarya-TURKEY.

E-MAIL: apirdal@sakarya.edu.tr

ORCID ID:0000-0002-2686-6043 
Mehmet Ali GÜNGÖR

AdDRESS: Sakarya University, Dept. of Mathematics, 54187, Sakarya-TURKEY. E-MAIL: agungor@sakarya.edu.tr

ORCID ID:0000-0003-1863-3183 